Pacific Journal of Mathematics

ON SOME COMPLETENESSES OF THE BERGMAN KERNEL 


\title{
ON SOME COMPLETENESSES OF THE BERGMAN KERNEL AND THE RUDIN KERNEL
}

\author{
Saburou SaItoh
}

Dedicated to Professor Toshio Umezawa on his 60-th birthday

Let $L_{2}(G)$ denote the Hilbert space of analytic functions $f$ which are regular in a region $G$ and have finite norms: $\left(\iint_{G}|f(z)|^{2} d x d y\right)^{1 / 2}<\infty$. It is well-known that the set $\left\{K\left(z, \bar{z}_{1}\right) \mid z_{1} \in G\right\}$ of the Bergman kernels for the class $L_{2}(G)$ is complete in $L_{2}(G)$. In this paper, for regular regions $G$ in the plane, it is shown that the set $\left\{K\left(z, \bar{z}_{1}\right) \mid z_{1} \in G\right\}$ is also complete in the Hilbert space of analytic functions $f$ which are regular in $G$ and finite norms: $\left(\int_{\partial G}|f(z)|^{2} d s\right)^{1 / 2}<\infty$.

The object of this paper is to discuss some problems of this type.

1. Introduction. Let $S$ be a compact bordered Riemann surface with contours $m$ and of genus $n$. Let $\left\{C_{\nu}\right\}_{\nu=1}^{2 n+m-1}$ denote a canonical homology basis and $\left\{C_{\nu}\right\}_{\nu=2 n+1}^{2 n+m}$ denote the boundary components. Let $M$ denote the Hilbert space of analytic differentials $f(z) d z$ which are regular in $S$ and have finite norms: $\left(\iint_{S}|f(z)|^{2} d x d y\right)^{1 / 2}<\infty(z=x+y i)$. Let $F=F\left(C_{j_{1}}, C_{j_{2}}, \cdots, C_{j_{a}}\right)$ be the closed subspace of $M$ composed of differentials $f(z) d z$ such that

$$
\int_{C_{\lambda_{\lambda}}} f(z) d z=0, \quad \lambda=1,2, \cdots, a .
$$

In terms of local parameters $z$ and $z_{1}$, let $K_{F}\left(z, \bar{z}_{1}\right) d z$ denote the Bergman kernel for the class $F$ which is characterized by the following reproducing property:

$$
f\left(z_{1}\right)=\iint_{S} f(z) \overline{K_{F}\left(z, \bar{z}_{1}\right)} d x d y \quad \text { for all } f(z) d z \in F
$$

On the other hand, we consider the Hilbert space $H_{2}^{D}$ of analytic differentials $f(z) d z$ which are regular in $S$ and finite norms: $\left(\frac{1}{2 \pi} \int_{\partial S}|f(z) d z|^{2} / i d W(z, t)\right)^{1 / 2}<\infty$. Here $W(z, t)$ denotes $g(z, t)+i g^{*}$ 
$(z, t), g$ is the Green function of $S$ with pole at $t$ and $g^{*}$ is the conjugate harmonic function of $g$. In this paper, for simplicity, we shall use the same notation for a point on $\bar{S}$ and a fixed local parameter around there. Let $H_{2}^{D F}$ denote the closed subspace of $H_{2}^{D}$ satisfying the condition (1.1). In terms of local parameters $z$ and $z_{1}$, let $\hat{R}_{t}^{F}\left(z, z_{1}\right) d z$ denote the conjugate Rudin kernel for the class $H_{2}^{D F}$ which is characterized by the following reproducing property (cf. [2]):

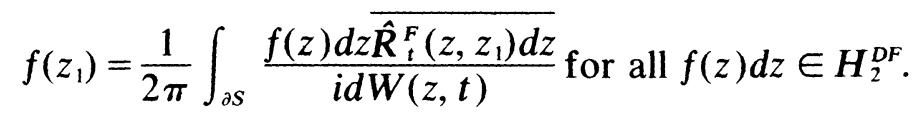

Let $S_{0}$ denote any point set $\left\{P_{\}}\right\}$of $S$ such that $\lim _{j \rightarrow \infty} P_{j}=P$, for some $P \in S$. Then as we see from the reproducing property, the sets of kernel functions $\left\{K_{F}\left(z, \bar{z}_{1}\right) d z \mid z_{1} \in S_{0}\right\}$ and $\left\{\hat{R}_{t}^{F}\left(z, z_{1}\right) d z \mid z_{1} \in S_{0}\right\}$ are complete (or equivalently closed) in the Hilbert spaces $\boldsymbol{F}$ and $\boldsymbol{H}_{2}^{D F}$, respectively. In the present paper, we shall show that the sets $\left\{K_{F}\left(z, \tilde{z}_{1}\right) d z \mid z_{1} \in S_{0}\right\}$ and $\left\{\hat{R}_{t}^{F}\left(z, z_{1}\right) d z \mid z_{1} \in S_{0}\right\}$ are also complete in $H_{2}^{D F}$ and $F$, respectively, and further we refer to some completenesses of the Rudin kernel functions. These results will be obtained from some fundamental properties of the Bergman kernel and the Rudin kernel.

2. Completeness of $\left\{K_{F}\left(z, \bar{z}_{1}\right) d z \mid z_{1} \in S_{0}\right\}$. Let

$$
L_{F}\left(z, z_{1}\right) d z
$$

denote the adjoint $L$-kernel of $K_{F}\left(z, \bar{z}_{1}\right) d z . \quad L_{F}\left(z, z_{1}\right) d z$ is an analytic differential on $\bar{S}$ except for $z_{1}$ where it has a double pole:

$$
L_{F}\left(z, z_{1}\right) d z=\left(\frac{1}{\pi} \frac{1}{\left(z-z_{1}\right)^{2}}+\text { regular terms }\right) d z
$$

Further $L_{F}\left(z, z_{1}\right) d z$ has the following properties:

$$
\begin{aligned}
& \int_{C_{l_{\lambda}}} L_{F}\left(z, z_{1}\right) d z=0, \quad \lambda=1,2, \cdots, a . \\
& \iint_{S} f(z) \overline{L_{F}\left(z, z_{1}\right)} d x d y=0 \text { for all } f(z) d z \in F . \\
& -K_{F}\left(z, \bar{z}_{1}\right) d z=\overline{L_{F}\left(z_{1}, z\right) d z} \text { along } \partial S(z \in \partial S) .
\end{aligned}
$$

In general, we have $K_{F}\left(z, \bar{z}_{1}\right)=\overline{K_{F}\left(z_{1}, \bar{z}\right)}$, but $L_{F}\left(z, z_{1}\right)=L_{F}\left(z_{1}, z\right)$ if and only if the class $F$ is symmetric. As to the properties of the Bergman 
kernel for the class $F$ on compact bordered Riemann surfaces, the reader is referred to Schiffer-Spencer [4]. Let $\left\{t_{\nu}\right\}_{\nu=1}^{2 n+m-1}$ denote the critical points of $g(z, t)$. Let $\left\{C_{k_{1}}, C_{k_{2}}, \cdots, C_{k_{b}}\right\}$ denote $\left\{C_{\nu}\right\}_{\nu=1}^{2 n+m-1}-$ $\left\{C_{j_{1}}, C_{j_{2}}, \cdots, C_{j_{a}}\right\}$. Then we have the following theorem which is a generalized form of Lemma 2.1 in [2]:

THEOREM 2.1 .

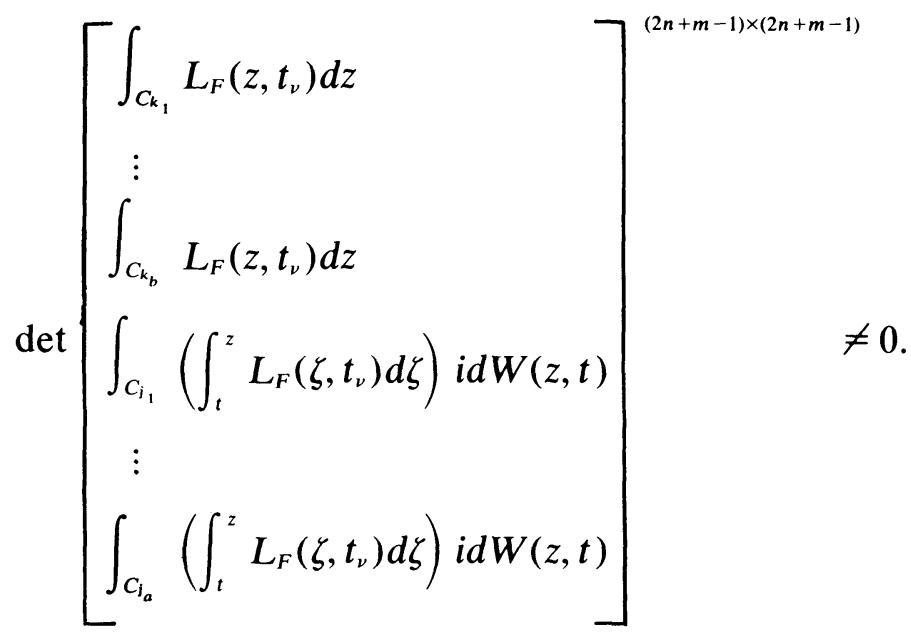

Here we assume that $\left\{t_{\nu}\right\}$ are all simple. In the other cases, we obtain modified forms.

Proof. From (2.1), (2.4) and the identity

$$
K_{F}\left(z_{1}, \bar{z}\right)=\frac{1}{2 \pi} \int_{\partial S} \frac{K_{F}(\zeta, \bar{z}) d \zeta \overline{\hat{R}_{t}^{F}\left(\zeta, z_{1}\right) d \zeta}}{i d W(\zeta, t)}
$$

we have

$$
\begin{aligned}
\overline{K_{F}\left(z_{1}, \bar{z}\right)} & =-\frac{1}{2 \pi i} \int_{\partial S} \frac{L_{F}(z, \zeta) \hat{R}_{t}^{F}\left(\zeta, z_{1}\right) d \zeta}{W^{\prime}(\zeta, t)} \\
& =-\frac{1}{\pi}\left(\frac{\hat{R}_{t}^{F}\left(z, z_{1}\right)}{W^{\prime}(z, t)}\right)^{\prime}-\sum_{\nu=1}^{2 n+m-1} \frac{\hat{R}_{t}^{F}\left(t_{\nu}, z_{1}\right) L_{F}\left(z, t_{\nu}\right)}{W^{\prime \prime}\left(t_{\nu}, t\right)}
\end{aligned}
$$

Hence

$$
\frac{1}{\pi}\left(\frac{\hat{R}_{t}^{F}\left(z, z_{1}\right)}{W^{\prime}(z, t)}\right)^{\prime}=-K_{F}\left(z, \bar{z}_{1}\right)-\sum_{\nu} \frac{\hat{R}_{t}^{F}\left(t_{\nu}, z_{1}\right)}{W^{\prime \prime}\left(t_{\nu}, t\right)} L_{F}\left(z, t_{\nu}\right)
$$




$$
\begin{gathered}
\frac{1}{\pi} \hat{R}_{t}^{F}\left(z, z_{1}\right) d z \\
=-\left\{\int_{t}^{z} K_{F}\left(\zeta, \bar{z}_{1}\right) d \zeta+\sum_{\nu} \frac{\hat{R}_{t}^{F}\left(t_{\nu}, z_{1}\right)}{W^{\prime \prime}\left(t_{\nu}, t\right)} \int_{t}^{z} L_{F}\left(\zeta, t_{\nu}\right) d \zeta\right\} d W(z, t) .
\end{gathered}
$$

At first from (2.5) we have

$$
\begin{gathered}
\sum_{\nu} \frac{\hat{R}_{t}^{F}\left(t_{\nu}, z_{1}\right)}{W^{\prime \prime}\left(t_{\nu}, t\right)} \int_{C_{k_{\mu}}} L_{F}\left(z, t_{\nu}\right) d z \\
=-\int_{C_{k_{\mu}}} K_{F}\left(z, \bar{z}_{1}\right) d z, \quad \mu=1,2, \cdots, b .
\end{gathered}
$$

Next from (2.6), since $\hat{R}_{t}^{F}\left(z, z_{1}\right) d z \in H_{2}^{D F}$, we have

$$
\begin{gathered}
\sum_{\nu} \frac{\hat{R}_{t}^{F}\left(t_{\nu}, z_{1}\right)}{W^{\prime \prime}\left(t_{\nu}, t\right)} \int_{C_{\lambda_{\lambda}}}\left(\int_{t}^{z} L_{F}\left(\zeta, t_{\nu}\right) d \zeta\right) i d W(z, t) \\
=-\int_{C_{\lambda_{\lambda}}}\left(\int_{t}^{z} K_{F}\left(\zeta, \bar{z}_{1}\right) d \zeta\right) i d W(z, t), \quad \lambda=1,2, \cdots, a .
\end{gathered}
$$

Here we shall see that the coefficients $\left\{\hat{R}_{t}^{F}\left(t_{\nu}, z_{1}\right) / W^{\prime \prime}\left(t_{v}, t\right)\right\}_{v}$ in the representation (2.6) of $\hat{R}_{t}^{F}\left(z, z_{1}\right) d z$ are determined uniquely as the solution of the equations (2.7) and (2.8).

We take $\left\{X_{\nu}\right\}_{\nu=1}^{2 m+m-1}$ as a solution of (2.7) and (2.8) and define

$$
\frac{1}{\pi} \tilde{R}_{t}^{F}\left(z, z_{1}\right) d z=-\left\{\int_{t}^{z} K_{F}\left(\zeta, \bar{z}_{1}\right) d \zeta+\sum_{\nu} X_{\nu} \int_{t}^{z} L_{F}\left(\zeta, t_{\nu}\right) d \zeta\right\} d W(z, t) .
$$

Then $\tilde{R}_{t}^{F}\left(z, z_{1}\right) d z \in H_{2}^{D F}$ and from (2.7) and (2.2) we see that $\left(K_{F}\left(\zeta, \bar{z}_{1}\right)+\right.$ $\left.\Sigma_{\nu} X_{\nu} L_{F}\left(\zeta, t_{\nu}\right)\right) d \zeta$ is exact. For any analytic differential $f(z) d z$ on $\bar{S}$ (in fact, $S$ ) such that $f(z) d z \in H_{2}^{D F}$, we have

$$
\begin{aligned}
& \frac{1}{2 \pi} \int_{\partial S} \frac{f(z) d z \overline{\tilde{R}_{t}^{F}\left(z, z_{1}\right) d z}}{i d W(z, t)} \\
= & \frac{1}{2 i} \int_{\partial S} f(z)\left(\overline{\int_{t}^{z}\left(K_{F}\left(\zeta, \bar{z}_{1}\right)+\sum_{\nu} X_{\nu} L_{F}\left(\zeta, t_{\nu}\right)\right) d \zeta}\right) d z,
\end{aligned}
$$

from the Green's formula, as usual,

$$
\left.=\iint_{S} f(z) \overline{\left(K_{F}\left(z, \bar{z}_{1}\right)+\sum_{\nu} X_{\nu} L_{F}\left(z, t_{\nu}\right)\right.}\right) d x d y
$$

from (2.3), 


$$
=f\left(z_{1}\right) \text {, }
$$

which implies that $\tilde{R}_{t}^{F}\left(z, z_{1}\right) \equiv \hat{R}_{t}^{F}\left(z, z_{1}\right)$. Since $\left\{\int_{t}^{z} L_{F}\left(\zeta, t_{\nu}\right) d \zeta\right\}$ is linearly independent, we have the desired result.

Thus from the uniqueness of the solution of the equations (2.7) and (2.8), we have the assertion of the theorem. In the cases of which all the $t_{\nu}$ are not simple, we can modify the above arguments slightly and we have modified forms, as usual.

In Theorem 2.1 , if $F=F(0)=M$, then from the identities

$$
L_{F}\left(z, z_{1}\right)=-\frac{2}{\pi} \frac{\partial^{2} g\left(z, z_{1}\right)}{\partial z \partial z_{1}} \text { and } Z_{\nu}^{\prime}(z)=-\int_{C_{\nu}} L_{F}(\zeta, z) d \zeta
$$

(cf. [4]), we have $\operatorname{det}\left[Z_{\nu}^{\prime}\left(t_{j}\right)\right]^{(2 n+m-1) \times(2 n+m-1)} \neq 0$, which is the result of Lemma 2.1 in [2]. Here $\left\{d Z_{\nu}\right\}$ is a basis of analytic differentials on $\bar{S}$ which are real along $\partial S$.

Next let $G$ be a regular region in the plane with contours $\left\{C_{\nu}\right\}_{\nu=1}^{m}$. If $F=F(1,2, \cdots, m-1)$, from the identities

$$
K_{F}\left(z, \bar{z}_{1}\right)=\frac{1}{\pi} M^{\prime}\left(z, z_{1}\right) \quad \text { and } \quad L_{F}\left(z, z_{1}\right)=-\frac{1}{\pi} N^{\prime}\left(z, z_{1}\right)
$$

(cf. [1], pp. 361-376), we have the following:

Corollary 2.1.

$$
\operatorname{det}\left[\frac{1}{2 \pi} \int_{C_{l}} N\left(z, t_{\nu}\right) i d W(z, t)-N\left(t, t_{\nu}\right) \omega_{j}(t)\right]^{(m-1) \times m-1)} \neq 0
$$

Here $\omega_{j}$ is the harmonic measure of $C_{j}$ and we assume that $\left\{t_{\nu}\right\}$ are all simple. In the other cases, we have modified forms.

Now we have the first desired result:

THEOREM 2.2. The set of the Bergman kernels $\left\{K_{F}\left(z, \bar{z}_{1}\right) d z \mid z_{1} \in\right.$ $\left.S_{0}\right\}$ is complete in $H_{2}^{D F}$.

Proof. We assume that for any $f(\zeta) d \zeta \in H_{2}^{D F}$,

$$
\int_{\partial S} \frac{f(\zeta) d \zeta \overline{K_{F}(\zeta, \bar{z}) d \zeta}}{i d W(\zeta, t)}=0 \text { for all } z \in S_{0}
$$

From (2.1) and (2.4), we have 


$$
\frac{1}{\pi}\left(\frac{f(z)}{W^{\prime}(z, t)}\right)^{\prime}+\sum_{\nu=1}^{2 n+m-1} \frac{f\left(t_{\nu}\right) L_{F}\left(z, t_{\nu}\right)}{W^{\prime \prime}\left(t_{\nu}, t\right)}=0 \text { for all } z \in S_{0}
$$

and hence for all $z \in S$. Here we assume that $\left\{t_{\nu}\right\}$ are all simple. At first from (2.10), we have

$$
\sum_{\nu} \frac{f\left(t_{\nu}\right)}{W^{\prime \prime}\left(t_{\nu}, t\right)} \int_{C_{k_{\mu}}} L_{F}\left(z, t_{\nu}\right) d z=0, \quad \mu=1,2, \cdots, b .
$$

Next from (2.10), we have

$$
\frac{1}{\pi} \frac{f(z)}{W^{\prime}(z, t)}+\sum_{\nu} \frac{f\left(t_{\nu}\right)}{W^{\prime \prime}\left(t_{\nu}, t\right)} \int_{t}^{z} L_{F}\left(\zeta, t_{\nu}\right) d \zeta=0 .
$$

Hence from $f(z) d z \in H_{2}^{D F}$, we get

$$
\begin{gathered}
\sum_{\nu} \frac{f\left(t_{\nu}\right)}{W^{\prime \prime}\left(t_{\nu}, t\right)} \int_{C_{\iota_{\lambda}}}\left(\int_{t}^{z} L_{F}\left(\zeta, t_{\nu}\right) d \zeta\right) i d W(z, t)=0, \\
\lambda=1,2, \cdots, a .
\end{gathered}
$$

Hence from (2.11), (2.13) and Theorem 2.1, we have $f\left(t_{\nu}\right)=0, \nu=$ $1,2, \cdots, 2 n+m-1$. Thus $\left(f(z) / W^{\prime}(z, t)\right)^{\prime} \equiv 0$ and $f(z) \equiv 0$. It implies the desired result.

In the cases of which all the $t_{\nu}$ are not simple, by making use of modified forms of Theorem 2.1, we have the desired result, again.

3. Completeness of $\left\{\hat{\boldsymbol{R}}_{t}^{F}\left(z, z_{1}\right) d z \mid z_{1} \in \boldsymbol{S}_{0}\right\}$. Let $N\left(z ; z_{1}, t\right)$ be a Neumann's function on $S$ with poles at $z_{1}$ and $t$, where $N\left(z ; z_{1}, t\right)+$ $\log \left|z-z_{1}\right|$ and $N\left(z ; z_{1}, t\right)-\log |z-t|$ are harmonic, respectively and $\partial N / \partial \nu=0$ on $\partial S$. We set $V\left(z ; z_{1}, t\right)=N\left(z ; z_{1}, t\right)+i N^{*}\left(z ; z_{1}, t\right)$ and define meromorphic differentials as follows:

$$
\begin{aligned}
& d P\left(z ; z_{1}, t\right)=\frac{1}{2}\left[d V\left(z ; z_{1}, t\right)-d W\left(z, z_{1}\right)+d W(z, t)\right] \\
& d \tilde{P}\left(z ; z_{1}, t\right)=\frac{1}{2}\left[d V\left(z ; z_{1}, t\right)-d W\left(z, z_{1}\right)-d W(z, t)\right] \\
& d Q\left(z ; z_{1}, t\right)=\frac{1}{2}\left[-d V\left(z ; z_{1}, t\right)-d W\left(z, z_{1}\right)+d W(z, t)\right] \\
& d \tilde{Q}\left(z ; z_{1}, t\right)=\frac{1}{2}\left[-d V\left(z ; z_{1}, t\right)-d W\left(z, z_{1}\right)-d W(z, t)\right] .
\end{aligned}
$$

Here we note that

$$
\overline{d P\left(z ; z_{1}, t\right)}=-d Q\left(z ; z_{1}, t\right) \text { along } \partial S,
$$




$$
\overline{d \tilde{P}\left(z ; z_{1}, t\right)}=-d \tilde{Q}\left(z ; z_{1}, t\right) \text { along } \quad \partial S .
$$

Then we have the following representation of the kernel $\hat{R}_{t}\left(z, z_{1}\right) d z$ for the class $H_{2}^{D}[2]$ :

$$
\hat{R}_{t}\left(z, z_{1}\right)=-\overline{W^{\prime}\left(z_{1}, t\right)} P^{\prime}\left(z ; z_{1}, t\right)+\sum_{\nu=1}^{2 n+m-1} \overline{\beta_{\nu}\left(z_{1}, t\right)} Z_{\nu}^{\prime}(z) .
$$

Here $\left\{\beta_{\nu}\left(z_{1}, t\right)\right\}$ are constants which depend on $z_{1}$ and $t$ and determined uniquely. At first, we note the following fact:

Lemma 3.1.

$$
\operatorname{det}\left[\beta_{\nu}\left(t_{\mu}, t\right)\right]^{(2 n+m-1) \times(2 n+m-1)} \neq 0 .
$$

Here we assume that $\left\{t_{\mu}\right\}$ are all simple. On the other cases, we have modified forms.

Proof. We assume that the determinant (3.5) is zero. Hence we can take complex numbers $\left\{X_{\mu}\right\}$ such that all $X_{\mu}$ are not zero and

$$
\sum_{\mu=1}^{2 n+m-1} X_{\mu} \beta_{\nu}\left(t_{\mu}, t\right)=0, \quad \nu=1,2, \cdots, 2 n+m-1 .
$$

On the other hand, from (3.4) we have

$$
\hat{R}_{t}\left(z, t_{\mu}\right)=\sum_{\nu=1}^{2 n+m-1} \overline{\beta_{\nu}\left(t_{\mu}, t\right)} Z_{\nu}^{\prime}(z), \quad \mu=1,2, \cdots, 2 n+m-1 .
$$

Hence from (3.6) and (3.7), we get

$$
\sum_{\mu} \bar{X}_{\mu} \hat{R}_{t}\left(z, t_{\mu}\right) \equiv 0
$$

As we see from the general theory of kernel functions, since $\operatorname{det}\left[\hat{R}_{t}\left(t_{\nu}, t_{\mu}\right)\right] \neq 0$, we have $X_{\mu}=0$ for all $\mu$ and hence we arrive at a contradiction.

Now we shall have the following theorem:

THEOREM 3.1.

$$
\operatorname{det}\left[\int_{C_{\lambda}}\left(\int_{C_{\mu}} \hat{R}_{t}\left(z, z_{1}\right) d z\right) \overline{d z_{1}}\right]^{(2 n+m-1) \times(2 n+m-1)}>0
$$


Proof. We assume that the determinant (3.8) is zero. Then by making use of the representation of $\hat{R}_{t}\left(z, z_{1}\right)$ by a complete orthonormal system, we see that $\left\{\int_{C_{\lambda}} \hat{R}_{t}\left(z, z_{1}\right) d z\right\}_{\lambda}$ is linearly dependent for any $z_{1} \in S$. Hence there exist complex numbers $\left\{X_{\lambda}\right\}$ such that all $X_{\lambda}$ are not zero and $\Sigma_{\lambda} X_{\lambda} \int_{C_{\lambda}} \hat{R}_{t}\left(z, z_{1}\right) d z \equiv 0$. As to this fact, the reader is referred to the proof of Theorem 2.1 in [3]. Hence from (3.4) we have $\sum_{\lambda} X_{\lambda} \int_{C_{\lambda}}\left(-\overline{W^{\prime}\left(z_{1}, t\right)} P^{\prime}\left(z ; z_{1}, t\right)+\sum_{\nu} \overline{\beta_{\nu}\left(z_{1}, t\right)} Z_{\nu}^{\prime}(z)\right) d z \equiv 0, \quad z_{1} \in S$.

By setting $z_{1}=t_{\mu}$, we have

$$
\sum_{\lambda} X_{\lambda}\left(\sum_{\nu} \overline{\beta_{\nu}\left(t_{\mu}, t\right)} \int_{C_{\lambda}} d Z_{\nu}\right)=0, \quad \mu=1,2, \cdots, 2 n+m-1 .
$$

Hence from Lemma 3.1 (or from modified forms of (3.5) if all the $t_{\nu}$ are not simple), we have $\Sigma_{\lambda} X_{\lambda} \int_{C_{\lambda}} d Z_{\nu}=0, \nu=1,2, \cdots, 2 n+m-1$, which implies that all the $X_{\lambda}$ are zero, because the matrix $\left\|\int_{C_{\lambda}} d Z_{\nu}\right\|$ is nonsingular. Thus we have a contradiction.

Next we consider a representation of $\hat{R}_{t}^{F}\left(z, z_{1}\right) d z$ by the kernel $\hat{R}_{t}\left(z, z_{1}\right) d z$. From Theorem 3.1, we can take constants $\left\{\hat{A}_{j_{\lambda}}\left(z_{1}\right)\right\}_{\lambda=1}^{a}$ which are analytic functions of $z_{1}$ and determined uniquely as follows:

$$
\hat{R}_{t}\left(z, z_{1}\right) d z-\frac{1}{2 \pi i} \sum_{\lambda=1}^{a} \overline{\hat{A}_{j_{\lambda}}\left(z_{1}\right)} \int_{C_{\lambda_{\lambda}}} \overline{\hat{R}_{t}(\zeta, z) d \zeta} d z \in H_{2}^{D F} .
$$

As we see by the simple computations, since the differential (3.9) has the reproducing property $(1.2)$, we see that this is the kernel $\hat{R}_{t}^{F}\left(z, z_{1}\right) d z$.

Now we shall give the following theorem:

THEOREM 3.2. For $\left\{\beta_{k_{\mu}}\left(z_{1}, t\right)\right\}_{\mu=1}^{b}$, we have

$$
\operatorname{det}\left[\begin{array}{c}
\beta_{k_{1}}\left(t_{\nu}, t\right) \\
\vdots \\
\beta_{k_{b}}\left(t_{\nu}, t\right) \\
\hat{A}_{j_{1}}\left(t_{\nu}\right) \\
\vdots \\
\hat{A}_{l a}\left(t_{\nu}\right)
\end{array}\right]^{(2 n+m-1) \times(2 n+m-1)} \neq 0
$$


Here we assume that $\left\{t_{\nu}\right\}$ are all simple. In the other cases, we obtain modified forms.

Proof. We assume that the above determinant is zero and hence we can take $\left\{Y_{\nu}\right\}$ such that all $Y_{\nu}$ are not zero and

$$
\begin{aligned}
& \sum_{\nu=1}^{2 n+m-1} Y_{\nu} \beta_{k_{\mu}}\left(t_{\nu}, t\right)=0, \quad \mu=1,2, \cdots, b, \quad \text { and } \\
& \sum_{\nu=1}^{2 n+m-1} Y_{\nu} \hat{A}_{\lambda_{\lambda}}\left(t_{\nu}\right)=0, \quad \lambda=1,2, \cdots, a .
\end{aligned}
$$

On the other hand, from (3.4) and (3.9) we have

$$
\begin{aligned}
\hat{R}_{t}^{F}\left(z, z_{1}\right)= & -\overline{W^{\prime}\left(z_{1}, t\right)} P^{\prime}\left(z ; z_{1}, t\right)+\sum_{\gamma} \overline{\beta_{\gamma}\left(z_{1}, t\right)} Z_{\gamma}^{\prime}(z) \\
& -\frac{1}{2 \pi i} \sum_{\lambda} \overline{\hat{A}_{j_{\lambda}}\left(z_{1}\right)} \int_{C_{I_{\lambda}}} \overline{\hat{R}_{t}(\zeta, z) d \zeta}
\end{aligned}
$$

Hence we have, by setting $z_{\mathrm{l}}=t_{\nu}$,

(3.12) $\hat{R}_{t}^{F}\left(z, t_{\nu}\right)=\sum_{\gamma} \overline{\beta_{\gamma}\left(t_{\nu}, t\right)} Z_{\gamma}^{\prime}(z)-\frac{1}{2 \pi i} \sum_{\lambda} \overline{\hat{A}_{j_{\lambda}}\left(t_{\nu}\right)} \int_{C_{\lambda_{\lambda}}} \overline{\hat{R}_{t}(\zeta, z) d \zeta}$.

From (3.10) and (3.12), we get

$$
\sum_{\nu} \bar{Y}_{\nu} \hat{R}_{t}^{F}\left(z, t_{\nu}\right)=\sum_{\lambda=1}^{a}\left(\sum_{\nu} \bar{Y}_{\nu} \overline{\beta_{j_{\lambda}}\left(t_{\nu}, t\right)}\right) Z_{j_{\lambda}}^{\prime}(z)
$$

and hence from $\hat{R}_{t}^{F}\left(z, t_{\nu}\right) d z \in H_{2}^{D F}$,

$$
\sum_{\lambda=1}^{a}\left(\sum_{\nu} \bar{Y}_{\nu} \overline{\beta_{j_{\lambda}}\left(t_{\nu}, t\right)}\right) \int_{C_{\lambda}} d Z_{j_{\lambda}}=0, \quad \lambda^{\prime}=1,2, \cdots, a .
$$

Since $\operatorname{det}\left[\int_{C_{i_{\lambda^{\prime}}}} d Z_{j_{\lambda}}\right] \neq 0$, we have

$$
\sum_{\nu} \bar{Y}_{\nu} \overline{\beta_{j_{\lambda}}\left(t_{\nu}, t\right)}=0, \quad \lambda=1,2, \cdots, a .
$$




$$
\sum_{\nu} \bar{Y}_{\nu} \hat{R}_{t}^{F}\left(z, t_{\nu}\right) \equiv 0
$$

which implies that all the $Y_{\nu}$ are zero. Hence we have a contradiction.

Especially, in Theorem 3.2, from the case of the subspace of $H_{2}^{D}$ such that $f(z) d z \in H_{2}^{D}$ are exact, we have the following:

Corollary 3.1 .

$$
\operatorname{det}\left[\hat{A}_{\nu}\left(t_{\mu}\right)\right]^{(2 n+m-1) \times(2 n+m-1)} \neq 0 .
$$

Here we assume that $\left\{t_{\mu}\right\}$ are all simple. On the other cases, we have modified forms.

Now we can give the second desired result:

THEOREM 3.3. The set of the conjugate Rudin kernels $\left\{\hat{R}_{t}^{F}\left(z, z_{1}\right) d z \mid z_{1} \in S_{0}\right\}$ is complete in $F$.

Proof. We assume that for any $f(z) d z \in F$,

$$
\iint_{S} f(z) \overline{\hat{R}_{t}^{F}\left(z, z_{1}\right)} d x d y=0 \text { for all } z_{1} \in S_{0} .
$$

From (3.4) and (3.9), we have

$$
\begin{gathered}
\iint_{S} f(z)\left[-W^{\prime}\left(z_{1}, t\right) \overline{P^{\prime}\left(z ; z_{1}, t\right)}+\sum_{\nu} \beta_{\nu}\left(z_{1}, t\right) \overline{Z_{\nu}^{\prime}(z)}\right] d x d y \\
+\frac{1}{2 \pi i} \sum_{\lambda} \hat{A}_{j_{\lambda}}\left(z_{1}\right) \iint_{S} f(z)\left(\int_{C_{\lambda_{\lambda}}} \hat{R}_{\mathrm{t}}(\zeta, z) d \zeta\right) d x d y \equiv 0 .
\end{gathered}
$$

Here since

$$
\iint_{S} f(z) \overline{Z_{\nu}^{\prime}(z)} d x d y=-\int_{C_{\nu}} f(z) d z \text { (cf. [4]), }
$$

from $f(z) d z \in F$, we have

$$
\begin{aligned}
& -W^{\prime}\left(z_{1}, t\right) \iint_{S} f(z) \overline{P^{\prime}\left(z ; z_{1}, t\right)} d x d y \\
& +\sum_{\mu=1}^{b} \beta_{k_{\mu}}\left(z_{1}, t\right)\left(-\int_{C_{k_{\mu}}} f(z) d z\right) \\
& +\frac{1}{2 \pi i} \sum_{\lambda=1}^{a} \hat{A}_{i_{\lambda}}\left(z_{1}\right) \iint_{S} f(z)\left(\int_{C_{\lambda_{\lambda}}} \hat{R}_{t}(\zeta, z) d \zeta\right) d x d y \equiv 0 .
\end{aligned}
$$


Here we assume that $\left\{t_{\nu}\right\}$ are all simple and we set $z_{1}=t_{\nu}$, in (3.14). Then from Theorem 3.2, we see that

$$
\iint_{S} f(z) \overline{P^{\prime}\left(z ; z_{1}, t\right)} d x d y=0 \text { for all } z_{1} \in S
$$

and $f(z) d z$ is exact. We set $\tilde{f}^{\prime}(z)=f(z)$ and from the Green's formula, we have

$$
\int_{\partial S} \tilde{f}(z) \overline{P^{\prime}\left(z ; z_{1}, t\right) d z} \equiv 0 .
$$

From (3.3), we have $\tilde{f}\left(z_{1}\right) \equiv \tilde{f}(t)$, which implies the desired result.

In the cases of which all the $t_{v}$ are not simple, by making use of modified forms of Theorem 3.2, we have the desired result, again.

4. Completeness of the Rudin kernel functions. Let $H_{2}$ denote the (analytic) Hardy class on $S$. Let $R_{t}\left(z, z_{1}\right)$ denote the Rudin kernel for the class $H_{2}$ which is characterized by the following reproducing property:

$$
f\left(z_{1}\right)=\frac{1}{2 \pi} \int_{\partial S} f(z) \overline{R_{t}\left(z, z_{1}\right)} i d W(z, t) \text { for all } f \in H_{2} .
$$

We shall consider the completenesses of the sets of differentials of $\left\{R_{t}\left(z, z_{1}\right) i d W(z, t) \mid z_{1} \in S_{0}\right\}$-type in $F$. Here we should consider the kernel $R_{t}^{F_{0}}\left(z, z_{1}\right)$ for the closed subspace $H_{2}^{F_{0}}$ of $H_{2}$ such that $f(z) i d W(z, t) \in H_{2}^{D F}$. We note that $R_{t}^{F_{0}}\left(z, z_{1}\right)$ is analytic on $\bar{S}$, as we see easily. At first we have the following fact:

THEOREM 4.1. The set of kernel functions $\left\{R_{t}^{F_{\mathrm{o}}}\left(z, z_{1}\right) \mid z_{1} \in S_{0}\right\}$ is complete in $\mathrm{H}_{2}^{F_{0}}$. The set of analytic differentials

$$
\left\{R_{t}^{F_{0}}\left(z, z_{1}\right) i d W(z, t) \mid z_{1} \in S_{0}\right\}
$$

is complete in $F$ if and only if $S$ is simply-connected.

Proof. The first part is evident, by the reproducing property. Next we assume that $S$ is not simply-connected. Then there exists at least one critical point $t^{*}$ of $g(z, t)$. We take $K_{F}\left(z, t^{*}\right)$ and we have, by the reproducing property of $K_{F}\left(z, t^{*}\right)$,

$$
\iint_{S} K_{F}\left(z, t^{*}\right) \overline{R_{t}^{F_{0}}\left(z, z_{1}\right) i W^{\prime}(z, t)} d x d y \equiv 0 \text { for all } z_{1} \in S
$$


Hence $\left\{R_{t}^{F_{o}}\left(z, z_{1}\right) i d W(z, t) \mid z_{1} \in S_{0}\right\}$ is not complete in $F$.

If $S$ is simply-connected, then we have the desired result, from the assertion of the next Theorem 4.2.

On the other hand, we consider the Rudin kernel $R_{t}^{F}\left(z, z_{1}\right)$ (with poles, in general) for the class $\boldsymbol{H}_{2}^{F}$ of meromorphic functions $f$ such that $f(z) i d W(z, t) \in H_{2}^{D F}$. Then we have the following identity, as we see by the simple computations,

$$
R_{t}^{F}\left(z, z_{1}\right) i d W(z, t) \overline{i d W\left(z_{1}, t\right)} \equiv \hat{R}_{t}^{F}\left(z, z_{1}\right) d z \overline{d z}_{1}
$$

Thus from (4.1) and Theorem 3.3, and from Theorem 2.2, we have the following theorem:

THEOREM 4.2. The set of differentials $\left\{R_{t}^{F}\left(z, z_{1}\right)\right.$ id $\left.W(z, t) \mid z_{1} \in S_{0}\right\}$ is complete in $F$. The set of meromorphic functions $\left\{K_{F}\left(z, \bar{z}_{1}\right) d z / i d W(z, t) \mid z_{1} \in S_{0}\right\}$ is complete in $H_{2}^{F}$.

In the last part, we shall give a representation of $R_{t}^{F}\left(z, z_{1}\right)$ by the kernel $\boldsymbol{R}_{\boldsymbol{t}}\left(z, z_{1}\right)$. At first we shall give the following theorem:

THEOREM 4.3 .

$$
\operatorname{det}\left[\int_{C_{\lambda}}\left(\int_{C_{\mu}} R_{t}\left(z, z_{1}\right) i d W(z, t)\right) \overline{i d W(z, t)}\right]^{(2 n+m) \times(2 n+m)}>0 .
$$

Proof. As we have pointed out in Theorem 3.1, it is sufficient to show that $\left\{\int_{C_{\lambda}} R_{t}\left(z, z_{1}\right) i d W(z, t)\right\}_{\lambda=1}^{2 n+m}$ is linearly independent. Suppose that

$$
\sum_{\lambda} X_{\lambda} \int_{C_{\lambda}} R_{t}\left(z, z_{1}\right) i d W(z, t) \equiv 0, \quad z_{1} \in S
$$

Here we use the following representation of $R_{t}\left(z, z_{1}\right) i d W(z, t)[2]$ :

(4.3) $R_{t}\left(z, z_{1}\right) i d W(z, t)=\left[-i \tilde{P}^{\prime}\left(z ; z_{1}, t\right)+\sum_{\nu=1}^{2 n+m-1} \overline{\alpha_{\nu}\left(z_{1}, t\right)} Z_{\nu}^{\prime}(z)\right] d z$.

Here $\left\{\alpha_{\nu}\left(z_{1}, t\right)\right\}$ are constants which depend on $z_{1}$ and $t$ and determined uniquely. From (4.2) and (4.3), we get

$$
\sum_{\lambda} X_{\lambda} \int_{C_{\lambda}}\left[-i \frac{\partial \tilde{P}^{\prime}\left(z ; z_{1}, t\right)}{\partial \bar{z}_{1}}+\sum_{\nu}\left(\overline{\frac{\partial \alpha_{\nu}\left(z_{1}, t\right)}{\partial z_{1}}}\right) Z_{\nu}^{\prime}(z)\right] d z \equiv 0
$$


We recall the following identities:

$$
\tilde{P}^{\prime}\left(z ; z_{1}, t\right)=\frac{\partial N\left(z ; z_{1}, t\right)}{\partial z}-\frac{\partial g\left(z, z_{1}\right)}{\partial z}-\frac{\partial g(z, t)}{\partial z},
$$

and

$$
\frac{\partial^{2} g\left(z, z_{1}\right)}{\partial z \partial \bar{z}_{1}}=\frac{\partial^{2} N\left(z ; z_{1}, t\right)}{\partial z \partial \bar{z}_{1}}-\frac{\pi}{2} \sum_{\mu, \nu=1}^{2 n+m-1} c_{\mu \nu} Z_{\mu}^{\prime}(z) \overline{Z_{\nu}^{\prime}\left(z_{1}\right)}
$$

Here the constants $c_{\mu \nu}$ are real, $c_{\mu \nu}=c_{\nu \mu}$ and the matrix $\left\|c_{\mu \nu}\right\|$ is nonsingular (cf. [4], p. 97). On the other hand, from (4.3) we have the following equations:

$$
\sum_{\nu} \overline{\alpha_{\nu}\left(z_{1}, t\right)} Z_{\nu}^{\prime}\left(t_{j}\right)=i \tilde{P}^{\prime}\left(t_{j} ; z_{1}, t\right), \quad j=1,2, \cdots, 2 n+m-1
$$

Here we assume that $\left\{t_{1}\right\}$ are all simple. On the other cases, we can modify the following arguments, as usual. Then since $\operatorname{det}\left[Z_{\nu}^{\prime}\left(t_{j}\right)\right] \neq 0$, we get

$$
\left(\overline{\frac{\partial \alpha_{\nu}\left(z_{1}, t\right)}{\partial z_{1}}}\right)=\left|\left(\frac{i \partial \tilde{P}^{\prime}\left(t_{j}^{\nu} ; z_{1}, t\right)}{\partial \bar{z}_{1}}\right)\right|^{\downarrow j} /\left|Z_{\nu}^{\prime}\left(t_{j}\right)\right| .
$$

Further we note that

$$
\int_{C_{\lambda}} K\left(z, \bar{z}_{1}\right) d z=-\iint_{S} K\left(z, \bar{z}_{1}\right) \overline{Z_{\lambda}^{\prime}(z)} d x d y=-\overline{Z_{\lambda}^{\prime}\left(z_{1}\right)}
$$

and we set $P_{\nu \lambda}=\int_{C_{\lambda}} d Z_{\nu}$.

Now from (4.4), (4.5), (4.6), (2.9), (4.7) and (4.8), we get

$$
\begin{gathered}
\pi i \sum_{\lambda} X_{\lambda} \overline{Z_{\lambda}^{\prime}\left(z_{1}\right)}+\frac{\pi i}{2} \sum_{\lambda} X_{\lambda}\left(\sum_{\mu, \nu} c_{\mu \nu} P_{\nu \lambda} \overline{Z_{\nu}^{\prime}\left(z_{1}\right)}\right) \\
+\sum_{\lambda} X_{\lambda}\left(\sum_{\nu}\left|i\left(\pi K\left(t_{j}, \bar{z}_{1}\right)-\frac{\pi}{2} \sum_{\mu, \nu} c_{\mu \nu} Z_{\mu}^{\prime}\left(t_{j}\right) \overline{Z_{\nu}^{\prime}\left(z_{1}\right)}\right)\right| \frac{P_{\nu \lambda}}{\left|Z_{\nu}^{\prime}\left(t_{j}\right)\right|}\right) \equiv 0 .
\end{gathered}
$$

Since in (4.9), each of the coefficients of $K\left(t_{j}, \bar{z}_{1}\right)$ must be zero, we compute the coefficients. Let $M_{\nu, j}$ denote the cofactor of the $(\nu, j)$ component of the matrix $\left\|Z_{\nu}^{\prime}\left(t_{j}\right)\right\|$. Then the coefficient of $\pi i K\left(t_{j}, \bar{z}_{1}\right) /\left|Z_{\nu}^{\prime}\left(t_{j}\right)\right|$ is given by 


$$
\begin{aligned}
& X_{1} P_{1,1} M_{1, j}+X_{1} P_{2,1} M_{2, j}+\cdots+X_{1} P_{2 n+m-1,1} M_{2 n+m-1, j} \\
+ & X_{2} P_{1,2} M_{1, j}+X_{2} P_{2,2} M_{2, j}+\cdots+X_{2} P_{2 n+m-1,2} M_{2 n+m-1, j} \\
& \ldots \\
& \ldots \\
+ & X_{2 n+m-1} P_{1,2 n+m-1} M_{, j}+\cdots \\
+ & X_{2 n+m-1} P_{2 n+m-1,2 n+m-1} M_{2 n+m-1, j} .
\end{aligned}
$$

Hence we have

$$
\begin{gathered}
\sum_{k=1}^{2 n+m-1} M_{k, j}\left(X_{1} P_{k, 1}+X_{2} P_{k, 2}+\cdots+X_{2 n+m-1} P_{k, 2 n+m-1}\right)=0 \\
j=1,2, \cdots, 2 n+m-1
\end{gathered}
$$

Since the matrix $\left\|M_{j, k}\right\|$ is the adjoint matrix of the regular matrix $\left\|Z_{\nu}^{\prime}\left(t_{j}\right)\right\|$, it is nonsingular. Hence we get $\Sigma_{\nu} X_{\nu} P_{k, \nu}=0$ for $k=$ $1,2, \cdots, 2 n+m-1$. Hence we have all the $X_{\nu}$ are zero, which implies the desired result.

Next we shall consider the class $\mathrm{H}_{2}^{g_{t}}$ of meromorphic functions $f$ such that $f(z) i d W(z, t)$ is analytic on $S$ except for $t$ and $f \in$ $L_{2}(\partial S)$. We shall construct the kernel $R_{2}^{g_{1}}\left(z, z_{1}\right)$ (with poles, in general) for the class $H_{2}^{g_{t}}$. In the following, without loss of generality, we assume that $\left\{t_{\nu}\right\}$ are all simple. Because in the other cases, we can modify the following arguments, slightly.

Let $L_{t}\left(z, z_{1}\right)$ and $\hat{L}_{t}\left(z, z_{1}\right)$ denote the adjoint $L$-kernels of $R_{t}\left(z, z_{1}\right)$ and $\hat{R}_{t}\left(z, z_{1}\right)$, respectively. They are analytic on $\bar{S}$ except for a simple pole at $z_{1}$ with residue 1 , and the following properties:

$$
\overline{R_{t}\left(z, z_{1}\right)} i d W(z, t)=\frac{1}{i} L_{t}\left(z, z_{1}\right) d z \quad \text { along } \quad \partial S, \quad \text { and }
$$

$$
\overline{\hat{R}_{t}\left(z, z_{1}\right)}=\frac{1}{i} \hat{L}_{t}\left(z, z_{1}\right) i d W(z, t) \text { along } \quad \partial S \text {. }
$$

respectively.

Further we have $\hat{L}_{t}\left(z, z_{1}\right)=-L_{t}\left(z_{1}, z\right)$ and

$$
L_{t}(z, t)=-\hat{L}_{t}(t, z)=-W^{\prime}(z, t)[2] .
$$

As we see by the simple computations, we have the following representation of $R_{t}^{g_{t}}\left(z, z_{1}\right)$ : 


$$
R_{t}^{g_{t}}\left(z, z_{1}\right) \equiv R_{t}\left(z, z_{1}\right)+\sum_{\nu=1}^{2 n+m-1} \overline{Y_{\nu}\left(z_{1}\right)} \hat{L}_{t}\left(z, t_{\nu}\right)
$$

Here $\left\{Y_{\nu}\left(z_{1}\right)\right\}$ are determined as the unique solution of the following equations:

$$
\sum_{\nu=1}^{2 n+m-1} Y_{\nu}\left(z_{1}\right) \hat{R}_{t}\left(t_{j}, t_{\nu}\right)=\hat{L}_{t}\left(z_{1}, t_{j}\right), \quad j=1,2, \cdots, 2 n+m-1
$$

Here we shall give the following theorem:

Theorem 4.4 .

$$
\operatorname{det}\left[\int_{C_{\lambda}}\left(\int_{C_{\mu}} R_{t}^{g_{t}}\left(z, z_{1}\right) i d W(z, t)\right) \overline{i d W\left(z_{1}, t\right)}\right]^{(2 n+m) \times(2 n+m)}>0 .
$$

Proof. Suppose that $\Sigma_{\lambda} X_{\lambda} \int_{C_{\lambda}} R_{t}^{g_{t}}\left(z, z_{1}\right) i d W(z, t) \equiv 0$ and hence

$$
\sum_{\lambda} X_{\lambda} \int_{C_{\lambda}} R_{t}\left(z, z_{1}\right) i d W(z, t)+\sum_{\lambda} X_{\lambda}\left(\sum_{\nu} \overline{Y_{\nu}\left(z_{1}\right)} \int_{C_{\lambda}} \hat{L}_{t}\left(z, t_{\nu}\right) i d W(z, t)\right)
$$

Since each $Y_{\nu}\left(z_{1}\right)$ is represented as a linear combination of $\left\{\hat{L}_{t}\left(z_{1}, t_{j}\right)\right\}_{j}$, we get

$$
\sum_{\lambda} X_{\lambda} \int_{C_{\lambda}} R_{t}\left(z, z_{1}\right) \operatorname{id} W(z, t) \equiv \sum_{\lambda} X_{\lambda}\left(\sum_{\nu} \overline{Y_{\nu}\left(z_{1}\right)} \int_{C_{\lambda}} \hat{L}_{t}\left(z, t_{\nu}\right) \operatorname{id} W(z, t)\right)
$$

Hence from Theorem 4.3, we have all the $X_{\lambda}$ are zero, which implies the desired result.

Now we construct the kernel $R_{t}^{F}\left(z, z_{1}\right)$. We set $C_{j_{0}}=\partial S$. Then from Theorem 4.4 , we have

$$
\begin{gathered}
\operatorname{det}\left[\int_{C_{i_{\lambda}}}\left(\int_{C_{i_{\lambda^{\prime}}}} R_{t}^{g_{t}}\left(z, z_{1}\right) i d W(z, t)\right) \overline{i d W\left(z_{1}, t\right)}\right]^{(a+1) \times(a+1)}>0, \\
\lambda, \lambda^{\prime}=0,1,2, \cdots, a .
\end{gathered}
$$

Hence we can take the unique constants $\left\{A_{j_{\lambda}}\left(z_{1}\right)\right\}_{\lambda=0}^{a}$ such that

$$
R_{t}^{g_{t}}\left(z, z_{1}\right)-\sum_{\lambda=0}^{a} \overline{A_{j_{\lambda}}\left(z_{1}\right)} \int_{C_{i_{\lambda}}} \overline{R_{t}^{g_{t}}(\zeta, z)} i d W(\zeta, t) \in H_{2}^{F},
$$


which is the kernel $R_{t}^{F}\left(z, z_{1}\right)$, as we see from the simple computations.

From (4.13) and (4.15), we have

$$
\begin{gathered}
R_{t}^{F}\left(z, z_{1}\right)=R_{t}\left(z, z_{1}\right)+\sum_{\nu=1}^{2 n+m-1} \overline{Y_{\nu}\left(z_{1}\right)} \hat{L}_{t}\left(z, t_{\nu}\right) \\
-\sum_{\lambda=0}^{a} \overline{A_{j_{\lambda}}\left(z_{1}\right)} \int_{C_{\lambda_{\lambda}}}\left[\overline{R_{t}(\zeta, z)}+\sum_{\nu=1}^{2 n+m-1} Y_{\nu}(z) \overline{\hat{L}_{t}\left(\zeta, t_{\nu}\right)}\right] \operatorname{idW}(\zeta, t) .
\end{gathered}
$$

Since $R_{t}^{F}\left(t, z_{1}\right)=0, \hat{L}_{t}\left(t, t_{\nu}\right)=0$ and $Y_{\nu}(t)=0$, as we see from (4.12) and (4.14), we have, by setting $z=t$ in (4.16),

$$
1-\sum_{\lambda=0}^{a} \overline{A_{\lambda_{\lambda}}\left(z_{1}\right)} \int_{C_{\lambda_{\lambda}}} i d W(\zeta, t)=0
$$

Hence we get (Note that the integral on $C_{\mathrm{j}}$ is zero.)

$$
\begin{gathered}
R_{t}^{F}\left(z, z_{1}\right)=\left(R_{t}\left(z, z_{1}\right)-1\right)+\sum_{\nu=1}^{2 n+m-1} \overline{Y_{\nu}\left(z_{1}\right)} \hat{L}_{t}\left(z, t_{\nu}\right) \\
-\sum_{\lambda=1}^{a} \overline{A_{\lambda_{\lambda}}\left(z_{1}\right)} \int_{C_{\lambda_{\lambda}}}\left[\overline{\left(R_{t}(\zeta, z)-1\right)}+\sum_{\nu=1}^{2 n+m-1} Y_{\nu}(z) \overline{\hat{L}_{t}\left(\zeta, t_{\nu}\right)}\right] \operatorname{id} W(\zeta, t) .
\end{gathered}
$$

\section{REFERENCES}

1. Z. Nehari, Conformal mapping, McGraw-Hill, 1952, 396 pp.

2. S. Saitoh, The kernel functions of Szegö type on Riemann surfaces, Kōdai Math. Sem. Rep., 24 (1972), 410-421.

3. - The weighted periods of analytic functions and the kernel functions of Szegö type for some closed subspaces, (to appear).

4. M. Schiffer and D. C. Spencer, Functionals of finite Riemann surfaces, Princeton (1954), 451 pp.

Received December 14, 1973.

Shibaura Institute of TECHNOLOGY 


\section{PACIFIC JOURNAL OF MATHEMATICS \\ EDITORS}

\author{
Richard ARENS (Managing Editor) \\ University of California \\ Los Angeles, California 90024
}

\author{
R. A. Beaumont \\ University of Washington \\ Seattle, Washington 98105
}

\section{J. DugundJI}

Department of Mathematics

University of Southern California

Los Angeles, California 90007

D. Gilbarg and J. Milgram

Stanford University

Stanford, California 94305

\section{ASSOCIATE EDITORS}
E. F. BECKENBACH
B. H. NeumanN
F. WOLF
K. YoshidA

\section{SUPPORTING INSTITUTIONS}

\author{
UNIVERSITY OF BRITISH COLUMBIA \\ CALIFORNIA INSTITUTE OF TECHNOLOGY \\ UNIVERSITY OF CALIFORNIA \\ MONTANA STATE UNIVERSITY \\ UNIVERSITY OF NEVADA \\ NEW MEXICO STATE UNIVERSITY \\ OREGON STATE UNIVERSITY \\ UNIVERSITY OF OREGON \\ OSAKA UNIVERSITY
}

\author{
UNIVERSITY OF SOUTHERN CALIFORNIA \\ STANFORD UNIVERSITY \\ UNIVERSITY OF TOKYO \\ UNIVERSITY OF UTAH \\ WASHINGTON STATE UNIVERSITY \\ UNIVERSITY OF WASHINGTON \\ AMERICAN MATHEMATICAL SOCIETY
}

The Supporting Institutions listed above contribute to the cost of publication of this Journal, but they are not owners or publishers and have no responsibility for its contents or policies.

Mathematical papers intended for publication in the Pacific Journal of Mathematics should be in typed form or offset-reproduced (not dittoed), double spaced with large margins. Underline Greek letters in red, German in green, and script in blue. The first paragraph or two must be capable of being used separately as a synopsis of the entire paper. Items of the bibliography should not be cited there unless absolutely necessary, in which case they must be identified by author and Journal, rather than by item number. Manuscripts, in duplicate, may be sent to any one of the four editors. Please classify according to the scheme of Math. Reviews, Index to Vol. 39. All other communications should be addressed to the managing editor, or Elaine Barth, University of California, Los Angeles, California, 90024.

100 reprints are provided free for each article, only if page charges have been substantially paid. Additional copies may be obtained at cost in multiples of 50 .

The Pacific Journal of Mathematics is issued monthly as of January 1966. Regular subscription rate: $\$ 72.00$ a year (6 Vols., 12 issues). Special rate: $\$ 36.00$ a year to individual members of supporting institutions.

Subscriptions, orders for back numbers, and changes of address should be sent to Pacific Journal of Mathematics, 103 Highland Boulevard, Berkeley, California, 94708.

PUBLISHED BY PACIFIC JOURNAL OF MATHEMATICS, A NON-PROFIT CORPORATION Printed at Jerusalem Academic Press, POB 2390, Jerusalem, Israel.

$$
\begin{gathered}
\text { Copyright (c) } 1975 \text { Pacific Journal of Mathematics } \\
\text { All Rights Reserved }
\end{gathered}
$$




\section{Pacific Journal of Mathematics}

Vol. 56, No. 2 December, 1975

Ralph Alexander, Generalized sums of distances .................. 297

Zvi Arad and George Isaac Glauberman, A characteristic subgroup of a group of odd order ............................... 305

B. Aupetit, Continuité du spectre dans les algèbres de Banach avec involution ........................................ 321

Roger W. Barnard and John Lawson Lewis, Coefficient bounds for some classes of starlike functions . ...........................

Roger W. Barnard and John Lawson Lewis, Subordination theorems for some classes of starlike fumctions . .................... 333

Ladislav Bican, Preradicals and injectivity .................. 367

James Donnell Buckholtz and Ken Shaw, Series expansions of analytic

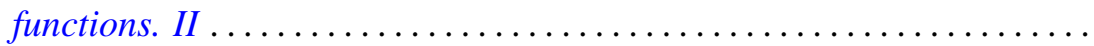

Richard D. Carmichael and E. O. Milton, Distributional boundary values in

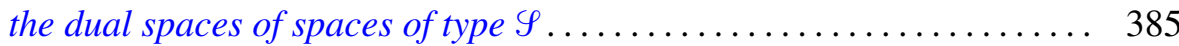

Edwin Duda, Weak-unicoherence ............................ 423

Albert Edrei, The Padé table of functions having a finite number of essential singularities ........................................

Joel N. Franklin and Solomon Wolf Golomb, A function-theoretic approach to the study of nonlinear recurring sequences ................ 455

George Isaac Glauberman, On Burnside's other $p^{a} q^{b}$ theorem 469

Arthur D. Grainger, Invariant subspaces of compact operators on topological vector spaces .........................

Jon Craig Helton, Mutual existence of sum and product integrals .

Franklin Takashi Iha, On boundary functionals and operators with finite-dimensional null spaces

Gerald J. Janusz, Generators for the Schur group of local and global number fields

A. Katsaras and Dar-Biau Liu, Integral representations of weakly compact operators.

W. J. Kim, On the first and the second conjugate points .

Charles Philip Lanski, Regularity and quotients in rings with involution ....

Ewing L. Lusk, An obstruction to extending isotopies of piecewise linear manifolds.

Saburou Saitoh, On some completenesses of the Bergman kernel and the Rudin kernel..

Stephen Jeffrey Willson, The converse to the Smith theorem for $Z_{p}$-homology spheres. 